\title{
Estimating Calorie Expenditure from Output Voltage of Piezoelectric Energy Harvester - an Experimental Feasibility Study
}

\author{
Guohao Lan ${ }^{1,2}$, Sara Khalifa ${ }^{1,2}$, Mahbub Hassan ${ }^{1,2}$, and $\mathrm{Wen} \mathrm{Hu}^{1,2}$ \\ ${ }^{1}$ School of Computer Science and Engineering, University of New South Wales, Sydney, NSW, Australia \\ ${ }^{2}$ National ICT Australia, Alexandria, NSW, Australia \\ \{glan, sarak, mahbub, wenh\}@cse.unsw.edu.au
}

\begin{abstract}
There is a growing interest in developing energy harvesting solutions for wearable devices so they can self-power themselves without relying on batteries. Piezoelectric energy harvesters (PEHs) can convert kinetic energy released from human activities into usable electrical energy for powering various electronic circuits inside the wearable device. Intuitively, the kinetic energy is produced because the user expends some calories during the physical activities. We therefore postulate that the voltage output of a $\mathrm{PEH}$ in a wearable device should contain information that can be used to estimate the amount of calorie expended. If this is true, then the PEH can be used as a new source for calorie estimation. Unlike conventional sensors, such as accelerometers, a $\mathrm{PEH}$ does not consume any power, which would make this new source very attractive. In this paper, using real $\mathrm{PEH}$ hardware and the data collected from ten real subjects, we conduct an experimental study to assess the suitability of $\mathrm{PEH}$ voltage in estimating calorie expenditure for two different activities, walking and running. We find that, for most subjects, the calorie estimations obtained from the output voltage of PEH is very close to those obtained from a 3-axial accelerometer.
\end{abstract}

\section{Categories and Subject Descriptors}

J.3 [Computer Applications]: Life and medical sciences

\section{General Terms}

Experimentation

\section{Keywords}

Piezoelectric Energy Harvester, Accelerometer, Calorie Expenditure Estimation

\section{INTRODUCTION}

Calorie expenditure estimation (CEE) is valuable in monitoring many health problems, such as obesity, an epidemic which is predicted to be the most preventive health problem in the future [25]. A large number of works in the literature have been devoted to estimate the calorie expenditure based on the output signals of accelerometers $[3,4,5,16]$ available in wearable devices. By combining acceleration data with anthropometric features, such as weight, height, age, etc., these algorithms can accurately estimate calorie expenditure of a user. These advancements have resulted in many popular wearable products in the market, such as Fitbit, Apple Watch, and Jawbone, that can collect and analyse human acceleration to estimate, display, or upload calorie expenditure on a continuous basis.

Accelerometers consume power. At the moment, most wearable devices are powered by batteries. There is, however, a growing interest in developing energy harvesting solutions for wearable devices so they can self-power themselves without relying on batteries [8, 17, 23, 24, 26]. Although there are several choices for energy harvesting, such as solar [10], wind [19], thermal [21], kinetic, etc., piezoelectric energy harvester $(\mathrm{PEH})$ is known to generate the most significant power by converting human motion to electricity [17]. If $\mathrm{PEH}$ is used, then it can be used to power the accelerometers and other circuits in a wearable device.

In this paper we seek to find a new use of PEH for CEE. Intuitively, the kinetic energy is produced because the user expends some calories during the physical activities. We therefore postulate that the voltage output of a $\mathrm{PEH}$ in a wearable device should contain information that can be used to estimate the amount of calorie expended. If this is true, then the PEH can be used as a new source for calorie estimation. Unlike conventional sensors used in CEE, such as accelerometers, a PEH does not consume any power, which would make this new source very attractive.

Using real PEH hardware and the data collected from ten real subjects, we conduct an experimental study to assess the suitability of PEH voltage in estimating calorie expenditure for two different activities, walking and running. We find that, for most subjects, the calorie estimations obtained 


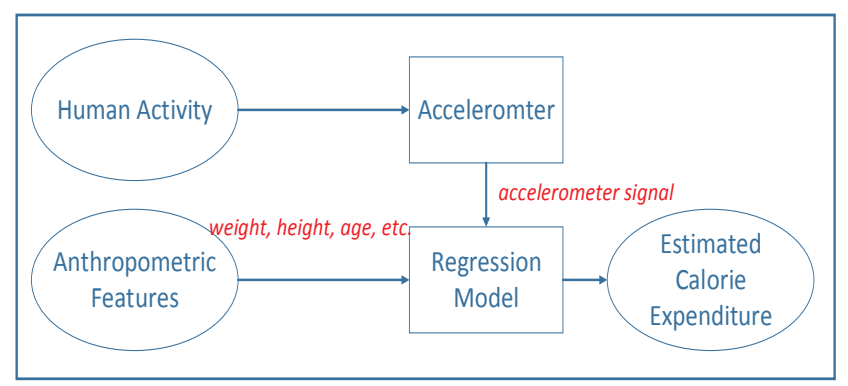

Figure 1: Architecture of the accelerometer based calorie expenditure estimation.

from the output voltage of PEH is very close to those obtained from a 3-axial accelerometer. The main contributions of this paper can be summarized as follows:

- We propose the use of output voltage of PEH as a new source of realising CEE for kinetic-powered devices, which is the first of its kind in the CEE literature.

- We propose a linear regression model based on PEH voltage data collected from ten subjects and show that, for most subjects, the calorie estimations obtained from the output voltage of $\mathrm{PEH}$ is very close to those obtained from a 3-axial accelerometer.

The rest of the paper is organised as follows. In Section 2, we explain the accelerometer-based CEE method we used in this study for comparison. We present our experimental hardware, our data collection and the proposed PEH-based CEE method in Section 3, followed by the performance evaluation in Section 4. Related work is reviewed in Section 5. We conclude our work in Section 6.

\section{ACCELEROMETER-BASED CEE}

Accelerometer based calorie expenditure estimation highly relies on the accelerometers to frequently sample the human activities. The architecture of the conventional accelerometer based CEE is shown in Figure 1. In order to estimate the calorie expenditure, accelerometer outputs are combined with the anthropometric features of the subjects to perform regression analysis $[4,5]$.

In this paper, we apply the popular method proposed in [4] to estimate the calorie expenditure base on the signal from a 3-axis accelerometer and the anthropometric features of the subjects. The regression model is given as follows:

$$
\overline{C E E}_{a c c}=a \times H(k)+b \times V(k)
$$

where, $\overline{C E E}_{a c c}$ represents the estimated calorie expenditure at the $k$ th minute. $H(k)$ was defined as the square root of the sum of squared of the accelerometer signals on the $x$ and $y$-axes $\left(H(k)=\sqrt{A c c_{x}^{2}+A c c_{y}^{2}}\right) . \quad V(k)$ was defined as the accelerometer signal on the $z$-axe $\left(V(k)=\sqrt{A c c_{z}^{2}}\right)$. Parameters $a$ and $b$ represent the coefficients of the regression model (1), and are generalized by stepwise multiple-linear regression based on the anthropometric features of 125 subjects in

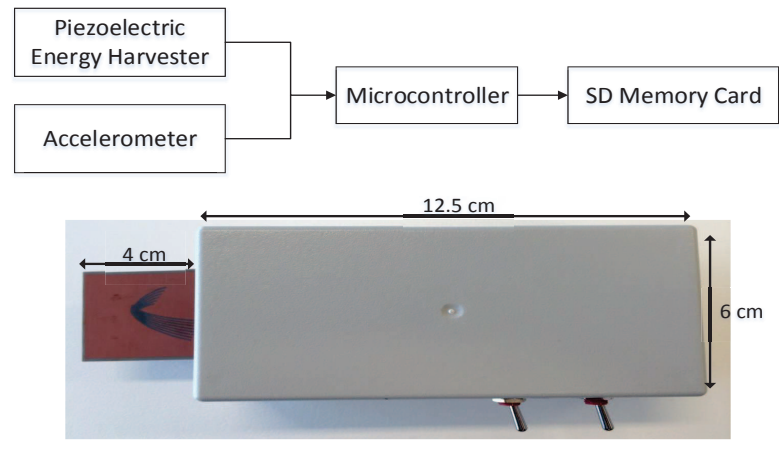

Figure 2: The hardware platform.

$$
\begin{aligned}
& \text { study [4]: } \\
& \qquad \begin{array}{c}
a=\frac{5.78 \times \text { Mass }+11.95 \times \text { Height }+6.89 \times \text { Age }-2001}{1000} \\
b=\frac{5.96 \times \text { Mass }+349.5}{1000}
\end{array}
\end{aligned}
$$

It has been reported that this regression model can achieve $60-95 \%$ correlation $[4,18]$ in estimating the calorie expenditure for walking and running.

\section{PEH-BASED CEE}

Pizoelectric energy harvester, which harvests energy from the vibration generated by the human motion, has been regarded as a promising energy source to power future wearable sensors. Unfortunately, a recent study [14] has shown that the amount of power that can be harvested from the energy harvester through the body motion can hardly be sufficient to power the accelerometers at a high sampling rate, thus, it results in poor accuracy for the upper layer applications, such as activity recognition and calorie expenditure estimation.

Instead of using the signals from the accelerometers to estimate the calorie expenditure, or using the energy harvested by the energy harvester to power the accelerometer, the primary idea of our method is to develop an regression model based on the output voltage signals from the pizoelectric energy harvester directly. Prior to presenting the proposed method, we introduce the hardware we designed for this study and our data collection method.

\subsection{Hardware setup}

We designed and built a data logger for this study. The data logger includes a vibration energy harvesting product from the MIDÈ Technology called Volture, which implements the transducer to provide AC voltage as its output. Our hardware also includes a 3-axis accelerometer (MMA7361LC) to record the acceleration signals, simultaneously with the voltage signal. An Arduino Uno has been used as a microcontroller device for sampling the data from both the Volture and the accelerometer. A sampling rate of $1 \mathrm{KHz}$ has been used for data collection. The sampled data has been saved on an $8 \mathrm{~GB}$ microSD card which has been equipped to the Arduino using microSD shield. A 9 volts battery has been 


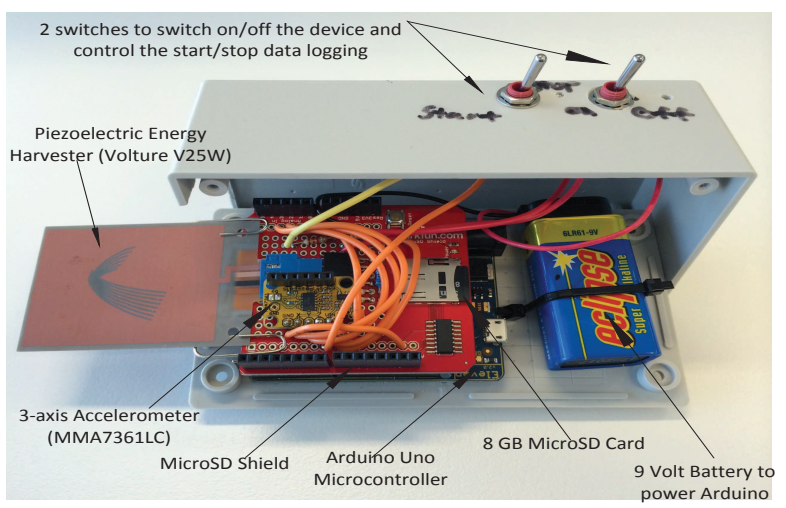

Figure 3: The internal appearance of the data logger.

used to power the Arduino. To control the data collection, our data logger also includes two switches, one to switch on/off the device and the other to control the start and stop of data logging. The hardware platform and the internal appearance of the data logger are shown in Figure 2 and Figure 3, respectively.

\subsection{Data collection}

Ten healthy subjects (4 male, and 6 female) of different ethnic backgrounds from our lab volunteered to participate in this research study. The participants were asked to hold the data logger in either their left or right hand and perform two different activities: walking and running. The details of the anthropometric features of the subjects are as follows: Age (26-35 years, $\mu=29$, and $\sigma=3.06)$, Weight (58-91 $\mathrm{Kg}, \mu=$ 69.3 , and $\sigma=10.21)$, Height $(154-185 \mathrm{~cm}, \mu=168.5$, and $\sigma$ $=9.98)$.

All subjects performed walking and running with their natural speed, i.e., there was no special speed control, thus, the step frequencies of walking and running vary among different subjects. To avoid mislabeling, the switch on the data logger has been used to control the start and stop of data collection at the beginning and end of each activity. Subjects were asked to stop and wait a few seconds after an activity and before starting the next activity. The data collected between the start and stop times of an activity were labeled with the name of that activity. Each subject provided between 25 and 35 seconds of data for both walking and running.

\subsection{Proposed Calorie Expenditure Estimation using Harvested Voltage Signal}

The architecture of the proposed pizoelectric energy harvester based method is shown in Figure 4. The system contains a kinetic energy harvester which harvests energy directly from human activity. Instead of using the harvested energy to power the accelerometer, the output voltage is used as the input signal, together with the subject's anthropometric features, to generate a regression model for calorie expenditure. No acceleration data is used in the proposed architecture comparing with the accelerometer based architecture given in Figure 1.

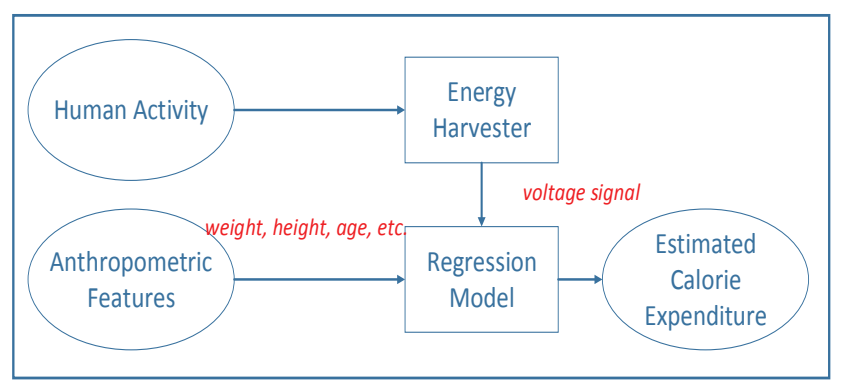

Figure 4: Architecture of the PEH based calorie expenditure estimation.

\subsection{Regression Model}

We performed a regression analysis to generate the generalized regression model. The linear regression model can be represented as follows:

$$
\overline{C E E}_{\text {volt }}=X \beta+\varepsilon
$$

where, $\overline{C E E}_{\text {volt }}$ indicates the estimated calorie expenditure at the $k$ th minute. $X$ denotes the vector of input signals, including the anthropometric features of the subjects (weight, height, and age), and the output voltage signals from the energy harvester. The $\beta$ and $\varepsilon$ are the vector of coefficients and residual error, respectively.

Without the ground truth from the indirect calorimeter, we applied the accelerometer based method proposed given in Equation (1) to estimate the calorie expenditure of the collected activity traces. The estimated results are used as the ground truth to train and calibrate our regression model. Note that, because Equation (1) is a generalized model, our regression model will not specify the activity type.

For each subject, $S_{i}$, in our subjects set, we have collected two acceleration signal traces, one for walking, and one for running. Using those two signal traces as inputs, we apply Equation (1) to get the acceleration based calorie expenditure estimations, $\overline{C E E}_{\text {acc_walk }}$, and $\overline{C E E}_{\text {acc_run }}$, for walking and running activity of subject $S_{i}$, respectively. These two acceleration based CEE results are synchronized with the recorded output voltage signal from the PEH during $S_{i}$ 's walking and running.

As an example, Figure 5 exhibits the output signals of the 3 -axis accelerometer for both walking and running of subject 2. Together with the anthropometric features of subject 2 , the acceleration signals are used to estimate the calorie expenditure (apply Equation (1), (2), and(3)). The results $\overline{C E E}_{a c c_{-} w a l k}$ and $\overline{C E E}_{a c c_{-} \text {run }}$ of subject 2 are given in Figure $6(\mathrm{a})$, which clearly indicates that running can expend more calories than walking. Correspondingly, Figure 6(b) shows the synchronized output voltage signals of the PEH recorded by our data logger for the same subject. Expectedly, PEH can harvest more energy from running than walking.

In order to generate the regression Equation (4), for each subject, $S_{i}$, we used the rest 9 subjects as the training set to train the linear regression model, and applied the Leaveone-out cross-validation to evaluate the performance of the 


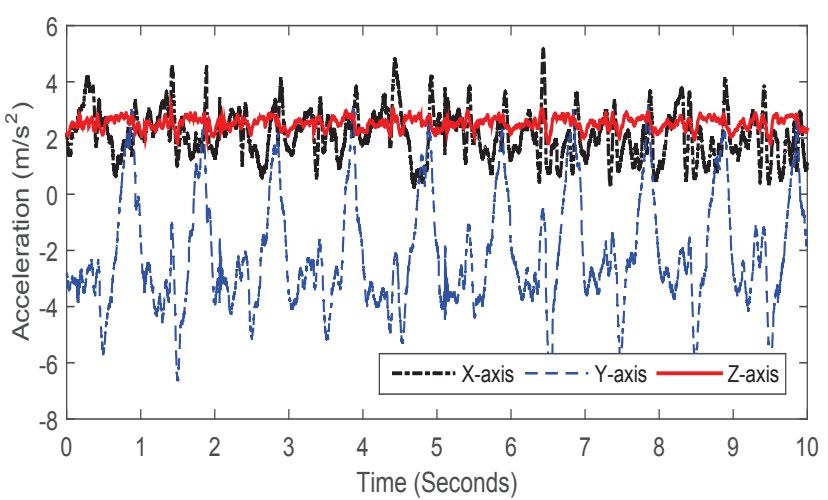

(a) Accelerometer Output for walking

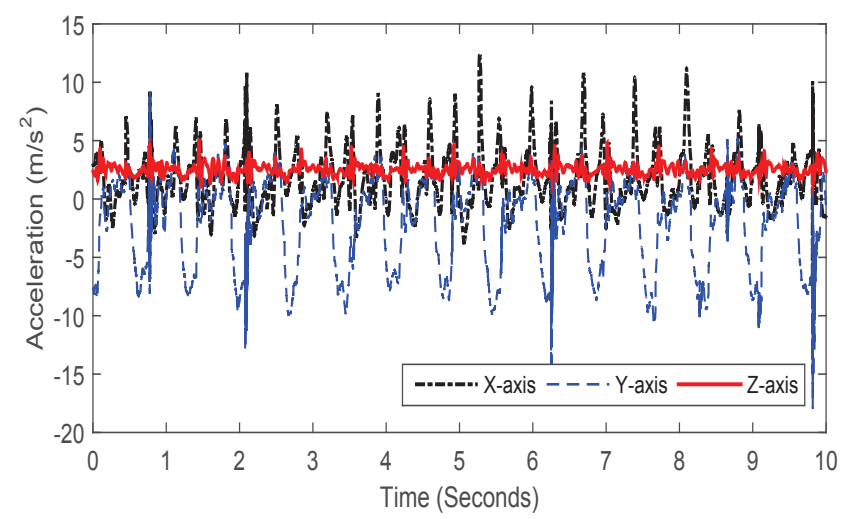

(b) Accelerometer Output for running

Figure 5: The output signal of the 3-axis accelerometer for both walking and running of subject 2 .

generated regression model on $S_{i}$. The Pearson's correlation coefficients of the linear correlation between the estimated calorie expenditure, $\overline{C E E}_{\text {volt }}$, and the input signal vector, $X$, for all the ten subjects are given in Figure 7. In case of walking, the Pearson's $r$ is 0.78 on average, and in case of running, we achieved a value of 0.71 on average, which indicates a strong positive correlation between the voltage signals and calorie expenditure for both walking and running.

\section{EXPERIMENTS RESULTS}

The instantaneous estimations of the PEH-based CEE and the ACC-based CEE, of walking and running, for all the ten subjects are given in Figure 8. Every second includes 1000 estimations. The results show that although the instantaneous estimations of our proposed $\mathrm{PEH}$-based method are different from that of the ACC-based method, the averages of the PEH-based CEE over a period of time (one second or longer), are very close to that of the ACC-based methods. The average value over longer terms is more useful in practical applications, comparing with the instantaneous measurements for every second. In addition, Figure 9 plots the mean of the estimated calorie expenditure over one second of the PEH-based and ACC-based methods. Figure 9(a) compares the $\overline{C E E}_{\text {volt }}$ of the PEH-based method with the $\overline{C E E_{a c c}}$ of the ACC-based methods for walking. The

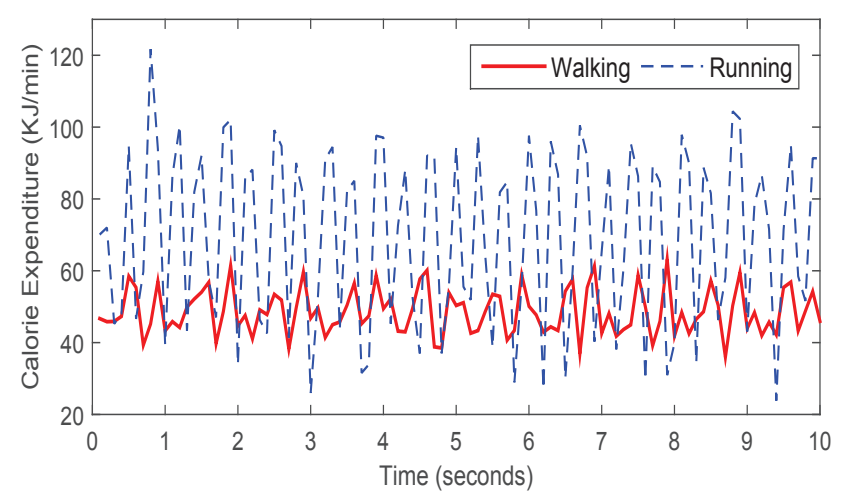

(a) Plot of the $\overline{C E E}_{a c c \_w a l k}$ and $\overline{C E E}_{\text {acc_run }}$ of subject 2, using the regression model given in Equation (1)

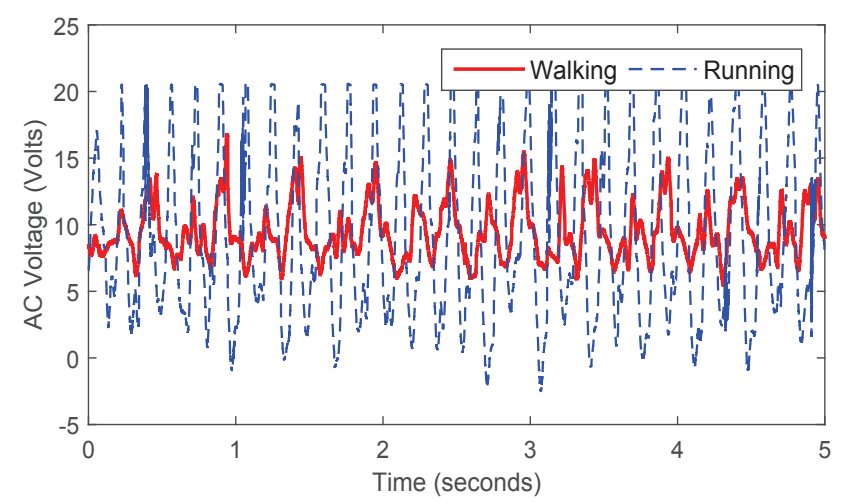

(b) Plot of the output voltage signals of pizoelectric energy harvester

Figure 6: Plot of the output voltage and calorie expenditure for subject 2 .

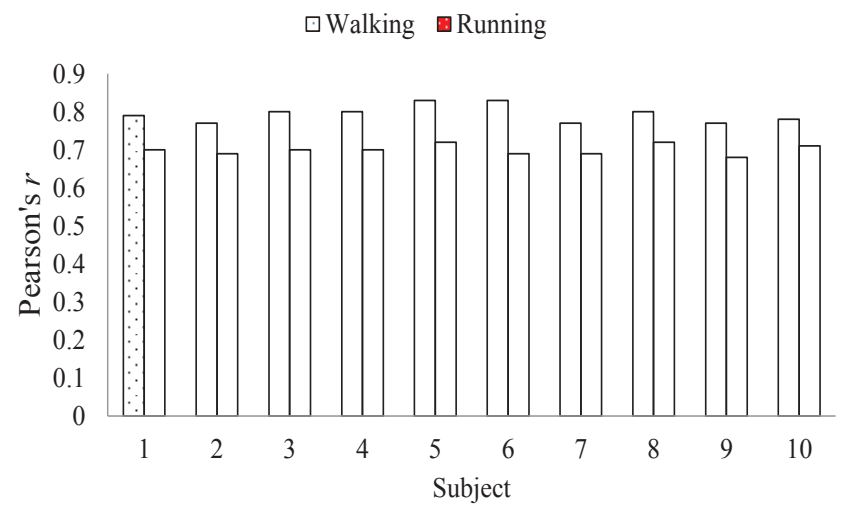

Figure 7: The Pearson's correlation coefficients of the linear correlation between the estimated results $\overline{C E E}_{\text {volt }}$ and the input signal vector $X$ for all ten subjects.

Mean Absolute Percentage Error (MAPE) is 0.12 in case of walking. Similarly, Figure 9(b) shows the results for running and the MAPE is 0.16 . However, which is indicated in Figure 8(o) and Figure 9(a), the PEH-based method will overestimate the calorie expenditure for walking. In addition, Figure $8(\mathrm{f})(\mathrm{l})(\mathrm{t})$ expose that the PEH-based method 


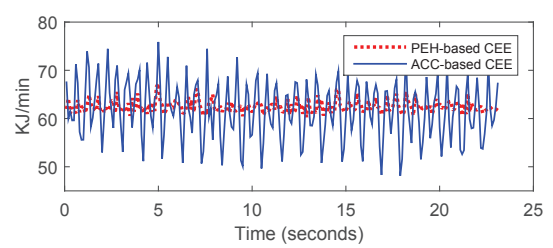

(a) subject1:walking

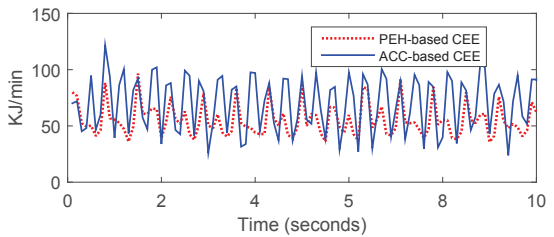

(d) subject2:running

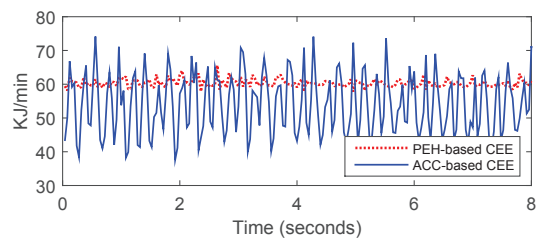

(g) subject4:walking

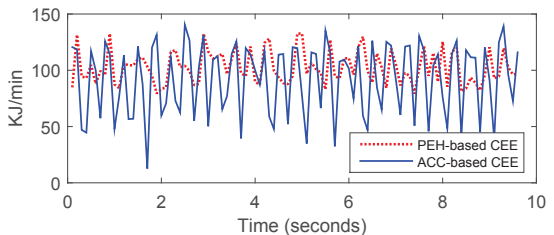

(j) subject5:running

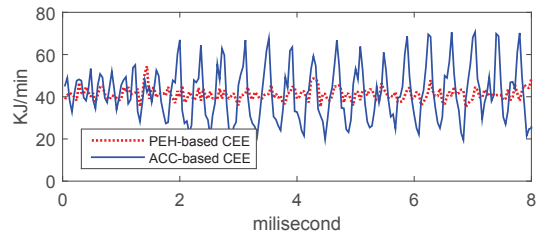

(m) subject7:walking

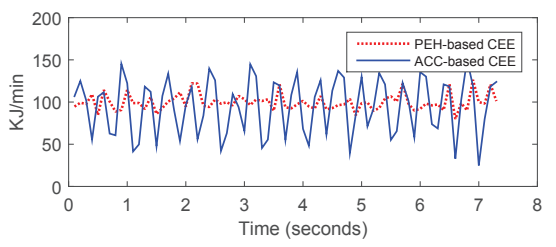

(p) subject8:running

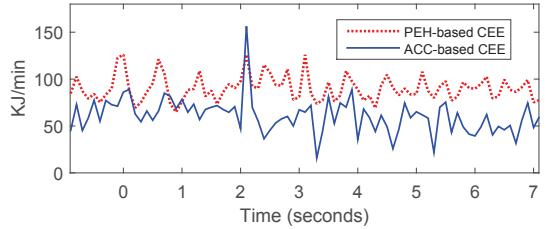

(b) subject1:running

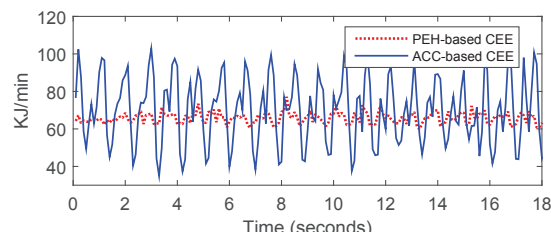

(e) subject3:walking

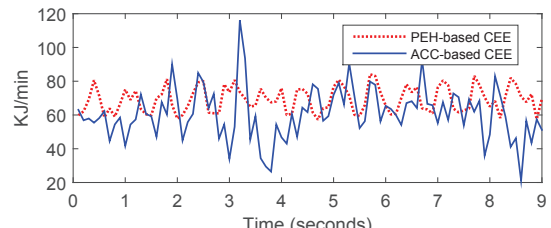

(h) subject4:running

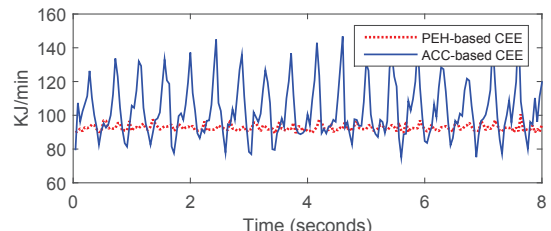

(k) subject6:walking

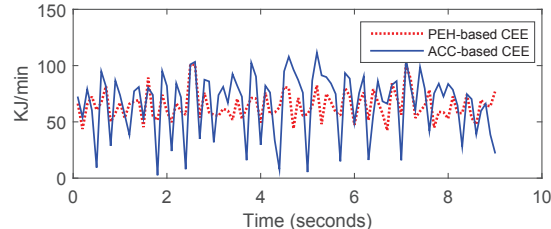

(n) subject7:running

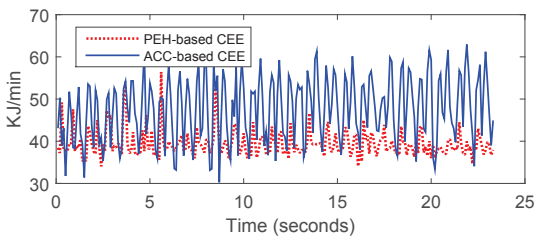

(q) subject9:walking

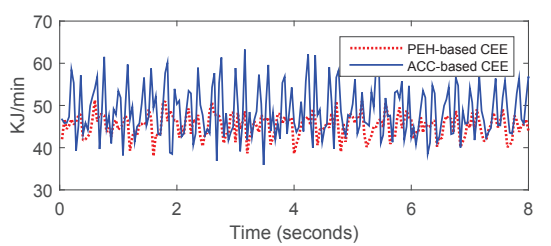

(c) subject2:walking

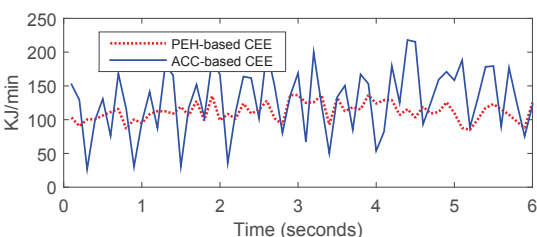

(f) subject3:running

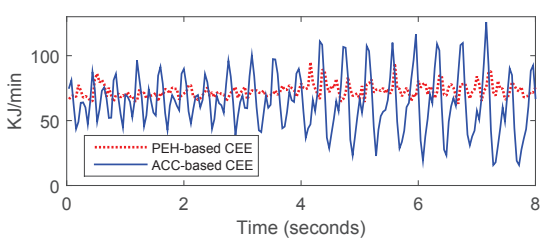

(i) subject5:walking

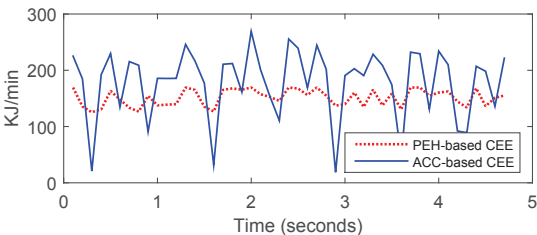

(1) subject6:running

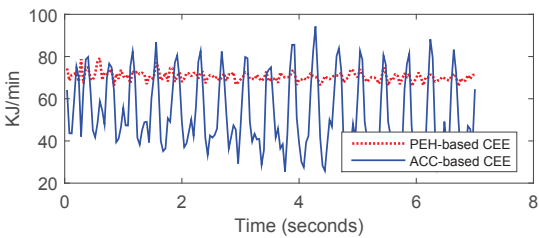

(o) subject8:walking

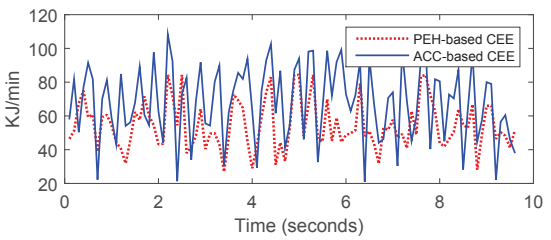

(r) subject9:running

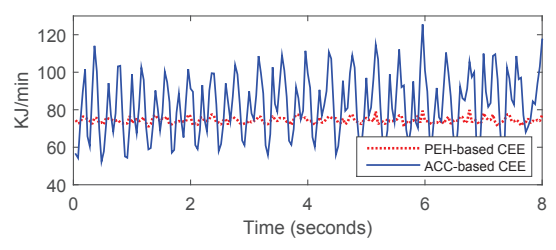

(s) subject10:walking

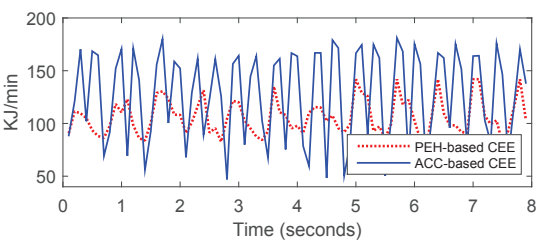

(t) subject10:running

Figure 8: The instantaneous estimation results of the PEH-based and ACC-based CEE for all the ten subjects, for both walking and running. 


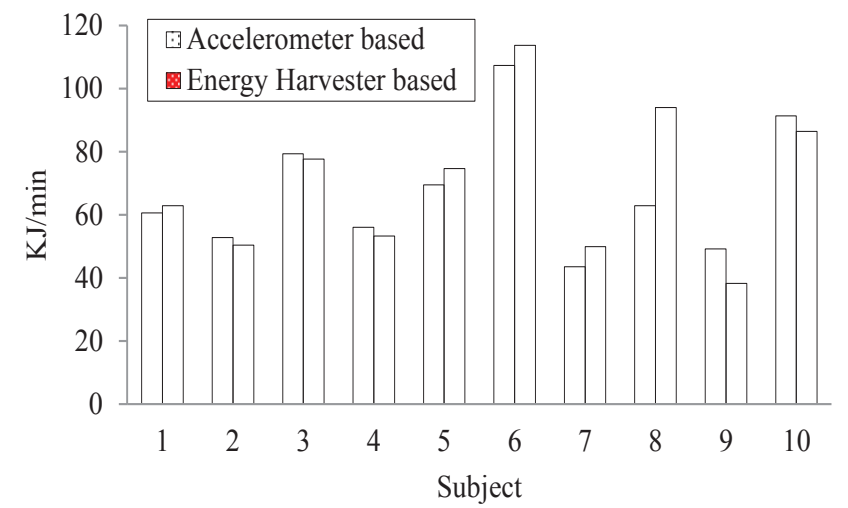

(a) Plot of the $\overline{C E E}_{v o l t}$ and $\overline{C E E}_{a c c}$ for walking

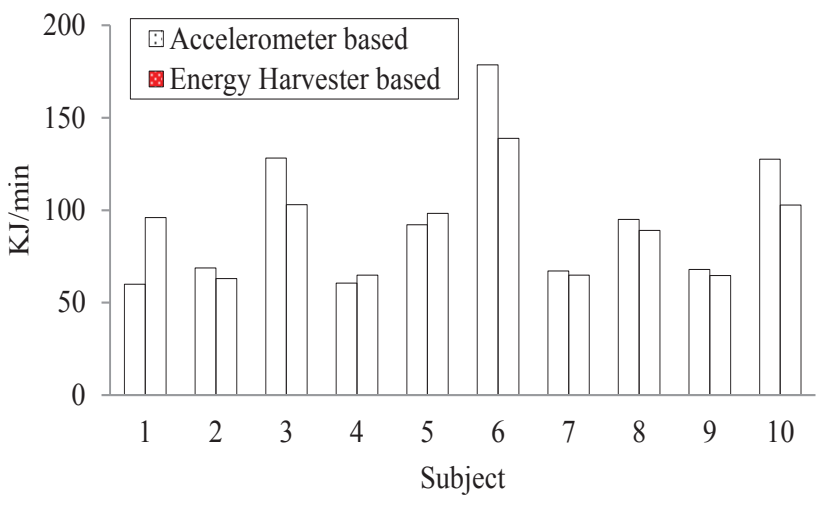

(b) Plot of the $\overline{C E E}_{v o l t}$ and $\overline{C E E}_{a c c}$ for running

Figure 9: The average CEE per second of the ACCbased and proposed PEH-based method.

will underestimate the calorie expenditure for running. We investigated that this results from the uncontrolled intensity and step frequency of the subjects' activity during our data collection. We will improve this by collecting more reliable data in our future work.

\section{RELATED WORK}

In the laboratory environment, the most accurate way of calorie expenditure estimation is to use the indirect calorimeters. Indirect calorimeter, such as the COSMED k4b2, estimates the amount of energy expenditure by measuring the consumption of oxygen and the production of carbon dioxide. Although it has been reported that using the indirect calorimeter can achieve a very high correlation with the actual calorie expenditure $[6,20]$, it is impractical for everyday calorie estimation, due to its inconvenience.

Outside the laboratory, wearable sensors are widely used as the alternatives to capture the information of daily activities and estimate the energy expenditure [1, 3, 5, 16, 18]. Early studies [7, 22] used accelerometer signals to generate a generalized regression model for the CEE of different activities. However, a study [1] has shown that, due to the variety of the activity intensities, methods using a single regression model will underestimate the expenditure of the high intensity activities, but overestimate that of the low intensity activities. To resolve this shortcoming, algorithms for human activity recognition (HAR), which have been widely used in the indoor positioning and smart living $[9,11,12,13]$, have also been applied in CEE. Recent studies have proposed the activity-specific CEE, which first recognizes the activity, and then generates an activity specific regression model [5], or assigns the Metabolic Equivalent of Task (MET, is a measurement of the calorie expenditure of a physical activity) to that activity $[1,2]$. Their results $[1,3]$ shown that the activity specific CEE methods can greatly improve the accuracy of CEE.

Unfortunately, the shortcoming of those methods is the power consumption of the wearable sensors, especially the accelerometers, used in the activity recognition is relatively high [14, 15]. To achieve continuous calorie estimation, we either need to instrument the wearable devices with large batteries, or frequently replace the batteries. Obviously, neither of these choices is desirable for future body area wearable devices. Compared to the literature, our work in this paper is unique in the sense that we do not use accelerometer at all to estimate calorie expenditure. PEH-based CEE is a totally new concept to the best of our knowledge. It opens the door to a completely new direction for calorie estimation. The fact that it can estimate calorie simply from the $\mathrm{PEH}$ data makes this method more competitive than the conventional accelerometer-based techniques for the realisation of battery-less wearables.

\section{CONCLUSION AND FUTURE WORK}

We have conducted the first experimental study to estimate calorie expenditure from the voltage output of PEH. We have used real PEH hardware and collected voltage data from 10 different subjects performing two different activities, walking and running. We have found that, for most subjects, the calorie estimations obtained from the output voltage of $\mathrm{PEH}$ is very close to those obtained from a 3-axial accelerometer. The result of this study shows the feasibility of estimation the calorie expenditure from the output signal of PEH in kinetic-powered wearable devices, which provides opportunities to develop new wearable devices for calorie estimation.

Motivated by the results of this paper, we plan to extend the current experimental study by collecting more data from a larger subject set, and studying a variety of activities with different intensities, including standing, walking, running, and biking. Moreover, instead of applying the estimated results from the accelerometer based method as the ground truth, we will use the indirect calorimeters, COSMED k4b2, to measure the actual calorie expenditure and train our regression model. Another direction of our future work would be generating activity-specific regression model for PEH based CEE. For activity recognition, we would like to apply the method proposed in [15] to achieve accelerometer-free activity recognition.

\section{ACKNOWLEDGEMENT}

We would like to thank all the participants for their help in the data collection. We also appreciate the anonymous reviewers for their detailed comments, which helped improving the final version of this paper.

\section{REFERENCES}


[1] F. Albinali, S. Intille, W. Haskell, and M. Rosenberger. Using wearable activity type detection to improve physical activity energy expenditure estimation. In Proceedings of the 12th ACM international conference on Ubiquitous computing, pages 311-320. ACM, 2010.

[2] M. Altini, J. Penders, and O. Amft. Energy expenditure estimation using wearable sensors: a new methodology for activity-specific models. In Proceedings of the conference on Wireless Health, page 1. ACM, 2012.

[3] M. Altini, J. Penders, R. Vullers, and O. Amft. Estimating energy expenditure using body-worn accelerometers: a comparison of methods, sensors number and positioning. Biomedical and Health Informatics, IEEE Journal of, 19(1):219-226, 2015.

[4] K. Y. Chen and M. Sun. Improving energy expenditure estimation by using a triaxial accelerometer. Journal of applied Physiology, 83(6):2112-2122, 1997.

[5] S. E. Crouter, K. G. Clowers, and D. R. Bassett. A novel method for using accelerometer data to predict energy expenditure. Journal of applied physiology, 100(4):1324-1331, 2006.

[6] R. Duffield, B. Dawson, H. Pinnington, and P. Wong. Accuracy and reliability of a cosmed k4b 2 portable gas analysis system. Journal of Science and Medicine in Sport, 7(1):11-22, 2004.

[7] P. S. Freedson, E. Melanson, and J. Sirard. Calibration of the computer science and applications, inc. accelerometer. Medicine and science in sports and exercise, 30(5):777-781, 1998.

[8] M. Gorlatova, J. Sarik, G. Grebla, M. Cong, I. Kymissis, and G. Zussman. Movers and shakers: Kinetic energy harvesting for the internet of things. In The 2014 ACM international conference on Measurement and modeling of computer systems, pages 407-419. ACM, 2014.

[9] M. Hassan. A performance model of pedestrian dead reckoning with activity-based location updates. In Networks (ICON), 2012 18th IEEE International Conference on, pages 64-69. IEEE, 2012.

[10] X. Jiang, J. Polastre, and D. Culler. Perpetual environmentally powered sensor networks. In Information Processing in Sensor Networks, 2005. IPSN 2005. Fourth International Symposium on, pages 463-468. IEEE, 2005.

[11] S. Khalifa and M. Hassan. Evaluating mismatch probability of activity-based map matching in indoor positioning. In Indoor Positioning and Indoor Navigation (IPIN), 2012 International Conference on, pages 1-9. IEEE, 2012.

[12] S. Khalifa, M. Hassan, and A. Seneviratne. Adaptive pedestrian activity classification for indoor dead reckoning systems. In Indoor Positioning and Indoor Navigation (IPIN), 2013 International Conference on, pages 1-7. IEEE, 2013.

[13] S. Khalifa, M. Hassan, and A. Seneviratne. Feature selection for floor-changing activity recognition in multi-floor pedestrian navigation. In Mobile Computing and Ubiquitous Networking (ICMU), 2014 Seventh International Conference on, pages 1-6. IEEE, 2014.
[14] S. Khalifa, M. Hassan, and A. Seneviratne. Pervasive self-powered human activity recognition without the accelerometer. In IEEE International Conference on Pervasive Computing and Communications (PerCom). IEEE, 2015.

[15] S. Khalifa, M. Hassan, A. Seneviratne, and S. K. Das. Energy harvesting wearables for activity-aware services. Internet Computing, IEEE, 20(5):2-10, 2015.

[16] J. Lester, C. Hartung, L. Pina, R. Libby, G. Borriello, and G. Duncan. Validated caloric expenditure estimation using a single body-worn sensor. In Proceedings of the 11th international conference on Ubiquitous computing, pages 225-234. ACM, 2009.

[17] P. D. Mitcheson, E. M. Yeatman, G. K. Rao, A. S. Holmes, and T. C. Green. Energy harvesting from human and machine motion for wireless electronic devices. Proceedings of the IEEE, 96(9):1457-1486, 2008.

[18] A. Pande, Y. Zeng, A. K. Das, P. Mohapatra, S. Miyamoto, E. Seto, E. K. Henricson, and J. J. Han. Energy expenditure estimation with smartphone body sensors. In Proceedings of the 8th International Conference on Body Area Networks, pages 8-14. ICST, 2013.

[19] C. Park and P. H. Chou. Ambimax: Autonomous energy harvesting platform for multi-supply wireless sensor nodes. In Sensor and Ad Hoc Communications and Networks, 2006. SECON'06. 2006 3rd Annual IEEE Communications Society on, volume 1, pages 168-177. IEEE, 2006.

[20] H. C. Pinnington, P. Wong, J. Tay, D. Green, and B. Dawson. The level of accuracy and agreement in measures of $\mathrm{f}$ e o 2 , f e co 2 and vì $\breve{G}$ e between the cosmed k4b 2 portable, respiratory gas analysis system and a metabolic cart. Journal of Science and Medicine in Sport, 4(3):324-335, 2001.

[21] M. Strasser, R. Aigner, M. Franosch, and G. Wachutka. Miniaturized thermoelectric generators based on poly-si and poly-sige surface micromachining. Sensors and Actuators A: Physical, 97:535-542, 2002.

[22] A. M. Swartz, S. J. Strath, D. R. Bassett, W. L. O Brien, G. A. King, and B. E. Ainsworth. Estimation of energy expenditure using csa accelerometers at hip and wrist sites. Medicine and Science in Sports and Exercise, 32(9; SUPP/1):S450-S456, 2000.

[23] T. Von Büren, P. D. Mitcheson, T. C. Green, E. M. Yeatman, A. S. Holmes, and G. Tröster. Optimization of inertial micropower generators for human walking motion. Sensors Journal, IEEE, 6(1):28-38, 2006.

[24] R. Vullers, R. van Schaijk, I. Doms, C. Van Hoof, and R. Mertens. Micropower energy harvesting. Solid-State Electronics, 53(7):684-693, 2009.

[25] Y. Wang, M. A. Beydoun, L. Liang, B. Caballero, and S. K. Kumanyika. Will all americans become overweight or obese? estimating the progression and cost of the us obesity epidemic. Obesity, 16(10):2323-2330, 2008.

[26] J. Yun, S. N. Patel, M. S. Reynolds, and G. D. Abowd. Design and performance of an optimal inertial power harvester for human-powered devices. Mobile Computing, IEEE Transactions on, 10(5):669-683, 2011. 